\title{
Chaperoning of the histone octamer by the acidic domain of DNA repair factor APLF
}

Ivan Corbeski ${ }^{1,7}$, Xiaohu Guo ${ }^{2}$, Bruna V. Eckhardt ${ }^{3}$, Domenico Fasci ${ }^{4}$, Melissa A. Graewert ${ }^{5}$, Wouter Wiegant $^{6}$, Kees Vreeken ${ }^{6}$, Hans Wienk $^{1,2}$, Haico van Attikum ${ }^{6}$, Dmitri I. Svergun ${ }^{5}$, Albert J.R. Heck ${ }^{4}$, Rolf Boelens ${ }^{1}$, Titia K. Sixma ${ }^{2^{*}}$, Francesca Mattiroli ${ }^{3^{*}}$, Hugo van Ingen $^{1^{*}}$

${ }^{1}$ NMR Spectroscopy, Bijvoet Centre for Biomolecular Research, Utrecht University, Padualaan 8, $3584 \mathrm{CH}$ Utrecht, The Netherlands;

${ }^{2}$ Division of Biochemistry and Oncode Institute, The Netherlands Cancer Institute, Plesmanlaan 121, 1066 CX Amsterdam, The Netherlands;

${ }^{3}$ Hubrecht Institute, Uppsalalaan 8, 3584 CT Utrecht, The Netherlands;

${ }^{4}$ Biomolecular Mass Spectrometry and Proteomics, Bijvoet Centre for Biomolecular Research and Utrecht Institute for Pharmaceutical Sciences, Utrecht University, Padualaan 8, $3584 \mathrm{CH}$, Utrecht, The Netherlands;

${ }^{5}$ European Molecular Biology Laboratory (EMBL), Hamburg Unit, DESY, Notkestrasse 85, D-22607 Hamburg, Germany;

${ }^{6}$ Department of Human Genetics, Leiden University Medical Center, Einthoven weg 20, 2333 ZC Leiden, The Netherlands;

${ }^{7}$ Present address: Department of Biochemistry, University of Zurich, Winterthurerstrasse 190, CH-8057 Zurich, Switzerland;

correspondence to: h.vaningen@uu.nl correspondence may also be addressed to:

f.mattiroli@hubrecht.eu, t.sixma@nki.nl 
Nucleosome assembly requires the coordinated deposition of histone complexes H3-H4 and H2A-H2B to form a histone octamer on DNA. In the current paradigm, specific histone chaperones guide the deposition of first $\mathrm{H} 3-\mathrm{H} 4$ and then $\mathrm{H} 2 \mathrm{~A}-\mathrm{H} 2 \mathrm{~B}(1-5)$. Here, we show that the acidic domain of DNA repair factor APLF $\left(\mathrm{APLF}^{\mathrm{AD}}\right)$ can assemble the histone octamer in a single step, and deposit it on DNA to form nucleosomes. The crystal structure of the $\mathrm{APLF}^{\mathrm{AD}}$-histone octamer complex shows that $\mathrm{APLF}^{\mathrm{AD}}$ tethers the histones in their nucleosomal conformation. Mutations of key aromatic anchor residues in APLF $^{\mathrm{AD}}$ affect chaperone activity in vitro and in cells. Together, we propose that chaperoning of the histone octamer is a mechanism for histone chaperone function at sites where chromatin is temporarily disrupted.

APLF (Aprataxin and Polynucleotide kinase Like Factor) is a DNA repair factor in non-homologous end joining (NHEJ) repair of DNA double-strand breaks(6-8), a critical pathway involved in immune responses and cancer biology $(9,10)$. APLF contains a conserved $C$-terminal acidic domain (APLF ${ }^{A D}$ ) with histone chaperone function(11) (Fig. $1 \mathrm{~A}$ and Fig. S1). We recently found that $A P L F^{A D}$ is intrinsically disordered and can bind $\mathrm{H} 2 \mathrm{~A}-\mathrm{H} 2 \mathrm{~B}$ as well as $\mathrm{H} 3-\mathrm{H} 4$ with high affinity(12). Such promiscuous histone binding has been observed before for other histone chaperones(13-17) and argued to play a role in nucleosome assembly (18-20), but its structural basis and implications are not fully understood. We therefore wanted to understand how APLF interacts with H2A-H2B and H3$\mathrm{H} 4$ and determine its functional consequences.

We first wondered if $\mathrm{H} 2 \mathrm{~A}-\mathrm{H} 2 \mathrm{~B}$ and $\mathrm{H} 3-\mathrm{H} 4$ bind to the same or to different sites in APLF ${ }^{\mathrm{AD}}$. Using nuclear magnetic resonance (NMR) spectroscopy, we found that addition of either H2A-H2B or H3$\mathrm{H} 4$ to $A P L F^{A D}$ resulted in severe peak intensity losses for distinct groups of residues, showing that APLF $^{\mathrm{AD}}$ contains two non-overlapping, adjacent binding regions for H3-H4 (residues N460-E474) and H2A-H2B (residues Y476-E486) (Fig. 1B,C). Further NMR and isothermal titration calorimetry (ITC) experiments showed that $\mathrm{H} 3-\mathrm{H} 4$ binding involves the $\alpha 1-\alpha 2$ region of $\mathrm{H} 3$ and is unaffected by mutations of residues that are crucial for H2A-H2B binding and chaperone activity (Y476A/W485A, named double anchor mutant DA-AB)(12) (Fig. S2). We found however that mutation of Y462 in the $\mathrm{H} 3-\mathrm{H} 4$ binding region reduced binding affinity, indicating that $A P L F^{A D}$ has a distinct set of aromatic anchor residues to bind either $\mathrm{H} 2 \mathrm{~A}-\mathrm{H} 2 \mathrm{~B}$ or $\mathrm{H} 3-\mathrm{H} 4$ (Fig. S2). To test whether APLF ${ }^{\mathrm{AD}}$ could bind to both $\mathrm{H} 2 \mathrm{~A}-\mathrm{H} 2 \mathrm{~B}$ and $\mathrm{H} 3-\mathrm{H} 4$ simultaneously, we added $\mathrm{APLF}^{\mathrm{AD}}$ to a stoichiometric mixture of $\mathrm{H} 2 \mathrm{~A}-\mathrm{H} 2 \mathrm{~B}$ and $\mathrm{H} 3-\mathrm{H} 4$ (referred to as octamer-mix) and analyzed complex formation using size-exclusion chromatography (SEC) (Fig. 1D and Fig. S3). As expected, in the absence of other factors the mixture eluted as separate histone dimer and histone tetramer complexes. Strikingly, upon addition of 
$\mathrm{APLF}^{\mathrm{AD}}$ a single high-molecular weight complex was obtained that contained all three components: $\mathrm{APLF}^{\mathrm{AD}}, \mathrm{H} 2 \mathrm{~A}-\mathrm{H} 2 \mathrm{~B}$, and H3-H4 (Fig. S3). This suggested that two APLF ${ }^{\mathrm{AD}}$ may be able to chaperone (i.e., bind) all histone components of the nucleosome at once. To further confirm this, we used native mass spectrometry (MS) and observed formation of a complex of $123 \mathrm{kDa}$, corresponding to two APLF ${ }^{A D}$ bound to two copies of H2A-H2B and H3-H4 each, $\left(\mathrm{APLF}^{\mathrm{AD}}-\mathrm{H} 2 \mathrm{~A}-\mathrm{H} 2 \mathrm{~B}-\mathrm{H} 3-\mathrm{H} 4\right)_{2}$ (Fig. $1 \mathrm{E}$ and Fig. S4). This complex has overall globular shape as shown from small-angle X-ray scattering experiments (Fig. S5) and is formed with sub-micromolar affinity $\left(K_{\mathrm{D}} \sim 150 \mathrm{nM}\right)$ as measured from ITC experiments (Fig. S6). Overall, these data demonstrate that $\mathrm{APLF}^{\mathrm{AD}}$ can bind $\mathrm{H} 2 \mathrm{~A}-\mathrm{H} 2 \mathrm{~B}$ and $\mathrm{H} 3-\mathrm{H} 4$ simultaneously to form a stable and high-affinity complex that contains the core histones at the same stoichiometry as found in the nucleosome.

A

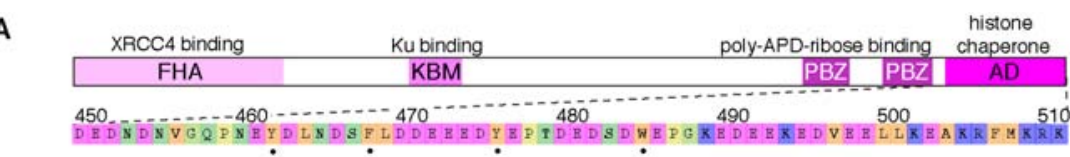

B

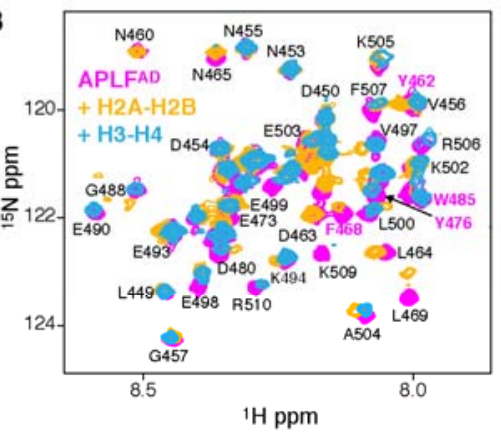

D

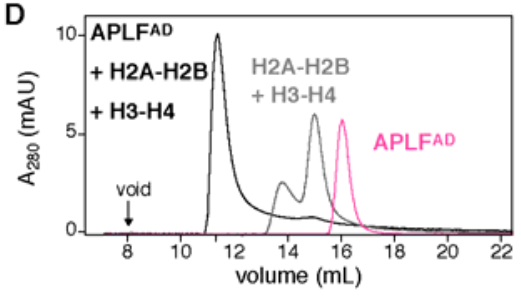

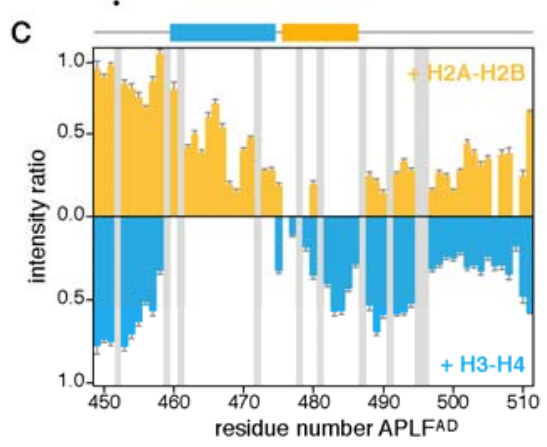

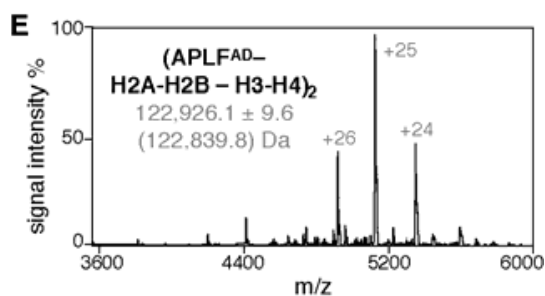

Fig. 1 | APLF ${ }^{\mathrm{AD}}$ binds H2A-H2B and H3-H4 to form a (APLF $\left.{ }^{\mathrm{AD}}-\mathrm{H} 2 \mathrm{~A}-\mathrm{H} 2 \mathrm{~B}-\mathrm{H} 3-\mathrm{H} 4\right)_{2}$ complex. A, APLF domain organization and $A P L F^{A D}$ sequence with color coding according to amino acid properties. Key residues for histone interactions $(\bullet)$ indicated. B, Overlaid NMR spectra of $A P L F^{A D}$, free and bound to $\mathrm{H} 2 \mathrm{~A}-\mathrm{H} 2 \mathrm{~B}$ or $\mathrm{H} 3-\mathrm{H} 4$. Aromatic residues labeled in magenta. C, Relative $\mathrm{APLF}^{\mathrm{AD}}$ NMR peak intensities upon binding $\mathrm{H} 2 \mathrm{~A}-\mathrm{H} 2 \mathrm{~B}$ or H3$\mathrm{H} 4$. Residues without data (prolines/overlapped peaks) in gray. The H2A-H2B (orange) and $\mathrm{H} 3-\mathrm{H} 4$ (blue) binding regions are indicated. D, SEC analysis of $\mathrm{APLF}^{\mathrm{AD}}$ and octamer-mix $(\mathrm{H} 2 \mathrm{~A}-\mathrm{H} 2 \mathrm{~B}+\mathrm{H} 3-\mathrm{H} 4)$ in absence and presence of saturating amounts of $\mathrm{APLF}^{\mathrm{AD}}$. E, Native-MS spectrum of the $\mathrm{APLF}^{\mathrm{AD}}$-histone complex with experimental (theoretical in brackets) molecular weights of the identified species. 
To understand how APLF chaperones core histones in the $\left(\mathrm{APLF}^{\mathrm{AD}}-\mathrm{H} 2 \mathrm{~A}-\mathrm{H} 2 \mathrm{~B}-\mathrm{H} 3-\mathrm{H} 4\right)_{2}$ complex, we set out to solve its structure. We obtained crystals of the complex reconstituted from tailless histones and a truncated $\mathrm{APLF}^{\mathrm{AD}}$ construct corresponding to residues $449-490\left(\mathrm{APLF}^{\mathrm{AD}-\Delta}\right.$ ). This truncation does not affect the binding affinity of $\operatorname{APLF}^{\mathrm{AD}}$ for histones (see Fig. $3 \mathrm{~A}$ below). We resolved the crystal structure of this complex at $2.35 \AA$ resolution (Fig. 2, Fig. S7 and Table S1). The histones $\mathrm{H} 2 \mathrm{~A}-\mathrm{H} 2 \mathrm{~B}$ and $\mathrm{H} 3-\mathrm{H} 4$ in the $\mathrm{APLF}^{\mathrm{AD}-\Delta}$-complex are arranged as in the nucleosome $(21,22)$ (0.56 $\AA$ backbone RMSD), involving histone-histone contacts across the $\mathrm{H} 3-\mathrm{H}^{\prime}$ ' tetramerization interface, the $\mathrm{H} 2 \mathrm{~B}-\mathrm{H} 4$ helical bundle, the $\mathrm{H} 2 \mathrm{~A}-\mathrm{H} 2 \mathrm{~A}^{\prime}$ interface and the $\mathrm{H} 2 \mathrm{~A}$ docking domain to $\mathrm{H} 3-\mathrm{H} 4$ (Fig. S8). The structure has overall pseudo two-fold symmetry, where two APLF ${ }^{A D}$ flank the octamer, tethering $\mathrm{H} 2 \mathrm{~A}-\mathrm{H} 2 \mathrm{~B}$ to $\mathrm{H} 3-\mathrm{H} 4$ within a histone half-octamer (Fig. 2A,B).

APLF $^{\mathrm{AD}}$ makes substantial interactions with both $\mathrm{H} 2 \mathrm{~A}-\mathrm{H} 2 \mathrm{~B}$ and $\mathrm{H} 3-\mathrm{H} 4$, covering $~ 800 \AA^{2}$ of histone surface on $\mathrm{H} 3-\mathrm{H} 4$ and $\sim 400 \AA^{2}$ on $\mathrm{H} 2 \mathrm{~A}-\mathrm{H} 2 \mathrm{~B}$ (Fig. $2 \mathrm{C}$ ). The regions involved in $\mathrm{H} 3-\mathrm{H} 4$ binding (residues P459-D471) and H2A-H2B binding (residues D482-P487) match well to the NMR results (Fig. 1C). The interaction is partially electrostatic, with an extensive network of intermolecular hydrogen bonds (Fig. 2C). Many of these interactions involve histone residues that otherwise bind the DNA phosphate backbone in the nucleosome, thus mimicking histone-DNA interactions (Fig. S9). In addition, aromatic residues of $\mathrm{APLF}^{\mathrm{AD}}$ provide anchors that make extensive van der Waals interactions with the histones. APLF residues Y462 and F468 protrude deeply into hydrophobic pockets on the $\mathrm{H} 3 \alpha 1-\alpha 2$-patch (Fig. 2D), consistent with their role in $\mathrm{H} 3-\mathrm{H} 4$ binding (Fig. S2). Similarly, APLF W485 anchors to a shallow hydrophobic pocket on the H2B $\alpha 1-\alpha 2-p a t c h$ (Fig. 2D), in line with its role in $\mathrm{H} 2 \mathrm{~A}-\mathrm{H} 2 \mathrm{~B}$ binding(12). Electron density for residues (E472-E481) that connect the $\mathrm{H} 2 \mathrm{~A}-\mathrm{H} 2 \mathrm{~B}$ and $\mathrm{H} 3-\mathrm{H} 4$ binding regions is incomplete or missing in all but one of the $\mathrm{APLF}^{\mathrm{AD}-\Delta}$ chains (Fig. S10), suggesting that this segment forms a flexible linker. Overall, APLF ${ }^{A D}$ makes use of multiple known histone dimer binding modes: the cap-anchor(23) and the R-finger interaction(24) for H2A$\mathrm{H} 2 \mathrm{~B}$, and the aromatic-key motif(25) for H3-H4 binding (Fig. 2C,D). Structural comparison to other histone chaperones (Fig. S11 and S12) shows that PPLF $^{A D}$ uniquely combines these binding modes to bind $\mathrm{H} 2 \mathrm{~A}-\mathrm{H} 2 \mathrm{~B}$ and $\mathrm{H} 3-\mathrm{H} 4$ simultaneously in their native histone octamer configuration.

$\mathrm{APLF}^{\mathrm{AD}}$ imposes both a steric and electrostatic block at the binding sites of both nucleosomal DNA gyres (Fig. 2B and S13). Additional NMR and intermolecular APLF ${ }^{A D}$-histone crosslinking MS (XL-MS) experiments suggest that the C-terminal residues 488-511, which are missing in the crystal structure, are in close proximity to the $\alpha \mathrm{C}$ helix of H2B on the octamer surface (Fig. S14), resulting in a model where $A P L F^{A D}$ envelops the histone octamer completely, except for the DNA binding region around the dyad (Fig. 2E and Fig. S14). 
A

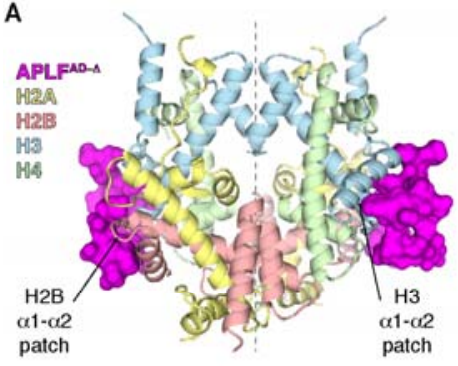

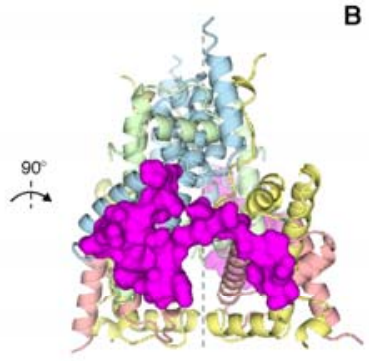

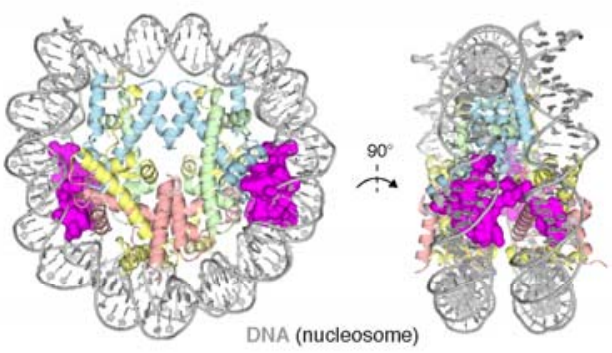

C

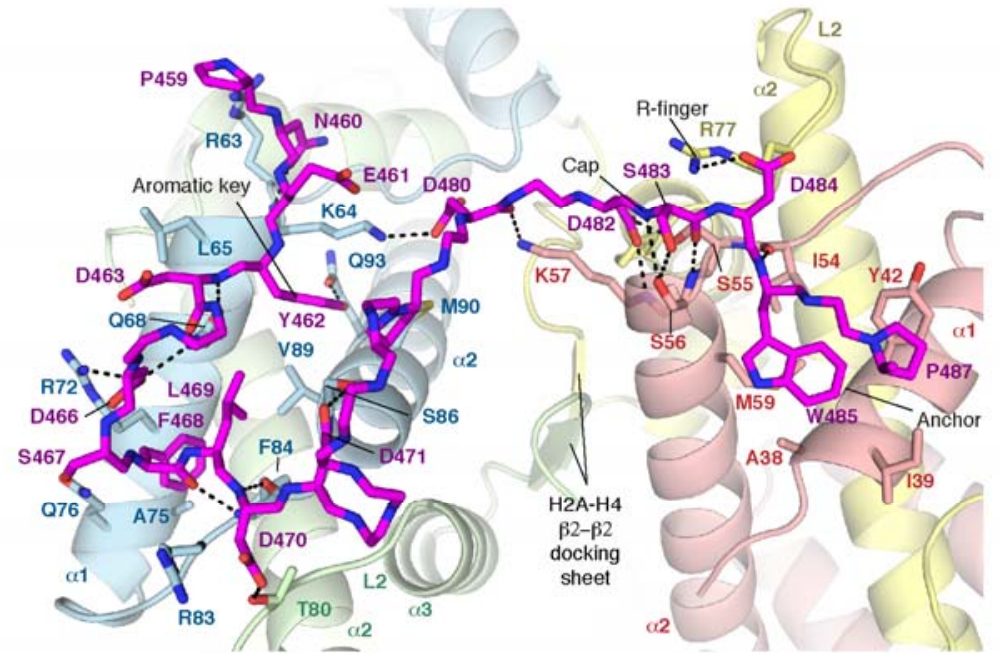

D
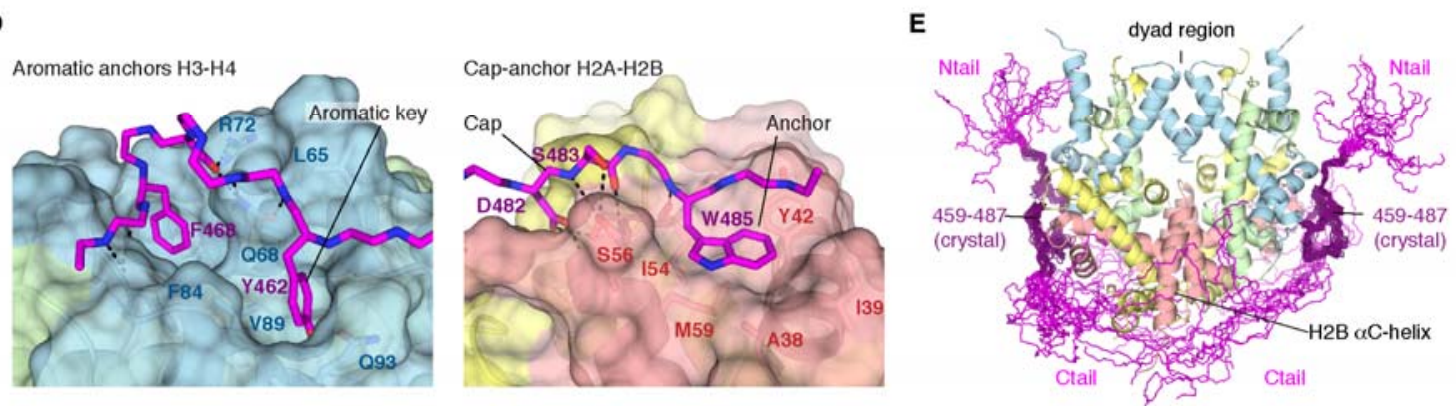

Fig. 2 | APLF ${ }^{A D-\Delta}$ binds H2A-H2B and H3-H4 as a histone octamer. A, Ribbon view of the crystal structure of the $\mathrm{APLF}^{\mathrm{AD}-\Delta}$-histone octamer complex with $\mathrm{APLF}^{\mathrm{AD}-\Delta}$ (APLF ${ }^{\mathrm{AD}}$ res. 449-490) shown as surface. Each APLF ${ }^{\mathrm{AD}-\Delta}$ binds primarily to the $\mathrm{H} 2 \mathrm{~B}$ and $\mathrm{H} 3 \alpha 1-\alpha 2$ patches (indicated) in each half-octamer. The pseudo-dyad axis is indicated with a dotted line. Color coding indicated in the Figure. B, Superposition of the APLF ${ }^{A D}-h_{i s t o n e}$ octamer complex and nucleosomal DNA highlighting the correspondence with the nucleosome structure and that APLF $^{\mathrm{AD}}$ blocks binding sites of both DNA gyres in the nucleosome. C, Zoom on the APLF ${ }^{\mathrm{AD}}$-histone octamer interface. Hydrogen bonds indicated as dashed lines; interface residues, the $\mathrm{H} 2 \mathrm{~A}-\mathrm{H} 4$ docking $\beta$-sheet and known histone dimer binding motifs are labeled. D, Zoom on the interaction of APLF ${ }^{A D}$ with H3-H4 (left) and $\mathrm{H} 2 \mathrm{~A}-\mathrm{H} 2 \mathrm{~B}$ (right). E, Superposition of the twenty best ranking models of the (APLF $\left.{ }^{\mathrm{AD}}-\mathrm{H} 2 \mathrm{~A}-\mathrm{H} 2 \mathrm{~B}-\mathrm{H} 3-\mathrm{H} 4\right)_{2}$ complex, where the APLF ${ }^{\mathrm{AD}} \mathrm{N}$ - and C-terminal tails are modeled based on NMR data and intermolecular crosslinks (see Fig. S14). 
To test the importance of the $\mathrm{H} 2 \mathrm{~A}-\mathrm{H} 2 \mathrm{~B}$ and $\mathrm{H} 3-\mathrm{H} 4$ interactions of $A P L F^{A D}$ for binding and chaperoning of the histone octamer, we mutated the aromatic anchor residues that are involved in binding based on the octamer complex structure or implicated in binding isolated $\mathrm{H} 2 \mathrm{~A}-\mathrm{H} 2 \mathrm{~B}(12)$ or H3-H4 (Fig. S2). We used double anchor mutants to disrupt either the H3-H4 interface (Y462A/F468A, DA-34) or the H2A-H2B interface (Y476A/W485A, DA-AB), and a quadruple anchor (QA) mutant that combines these mutations. Indeed, these mutations reduced the binding affinity of $\mathrm{APLF}^{\mathrm{AD}}$ to the octamer-mix up to five-fold (Fig. 3A). Additionally, removal of the anchor residues resulted in a large decrease of binding enthalpy, suggesting a reduction in buried surface and thus a defect in assembly of the histone octamer (Fig. S15). To further probe their importance for chaperone activity we tested wild-type and mutant $\mathrm{APLF}^{\mathrm{AD}}$ in their ability to prevent non-native histone-histone contacts using XL-MS. As expected, for the octamer-mix alone, most histone-histone cross-links obtained are incompatible with the histone octamer structure (Fig. 3B). Strikingly, addition of wild-type APLF ${ }^{A D}$, but not aromatic anchor mutants, dramatically reduced the number of incompatible cross-links, further substantiating that $\mathrm{APLF}^{\mathrm{AD}}$ functions as a histone chaperone and stabilizes the core histones in their nucleosomal octameric arrangement (Fig. 3B). To test if the aromatic anchor residues are also important for the cellular roles of APLF in NHEJ, we tested the effects of the QA mutant in human cells in response to DNA damage. Deletion of the acidic domain of APLF was previously found to abrogate histone binding in cells and to block the timely release of APLF from DNA damage sites(11). Consistent with these findings, we find that the QA mutation in APLF resulted in a slower release from DNA damage sites (Fig. 3C,D and Fig. S16). This underscores that APLF's chaperone activity is important for efficient progression of DNA repair. 

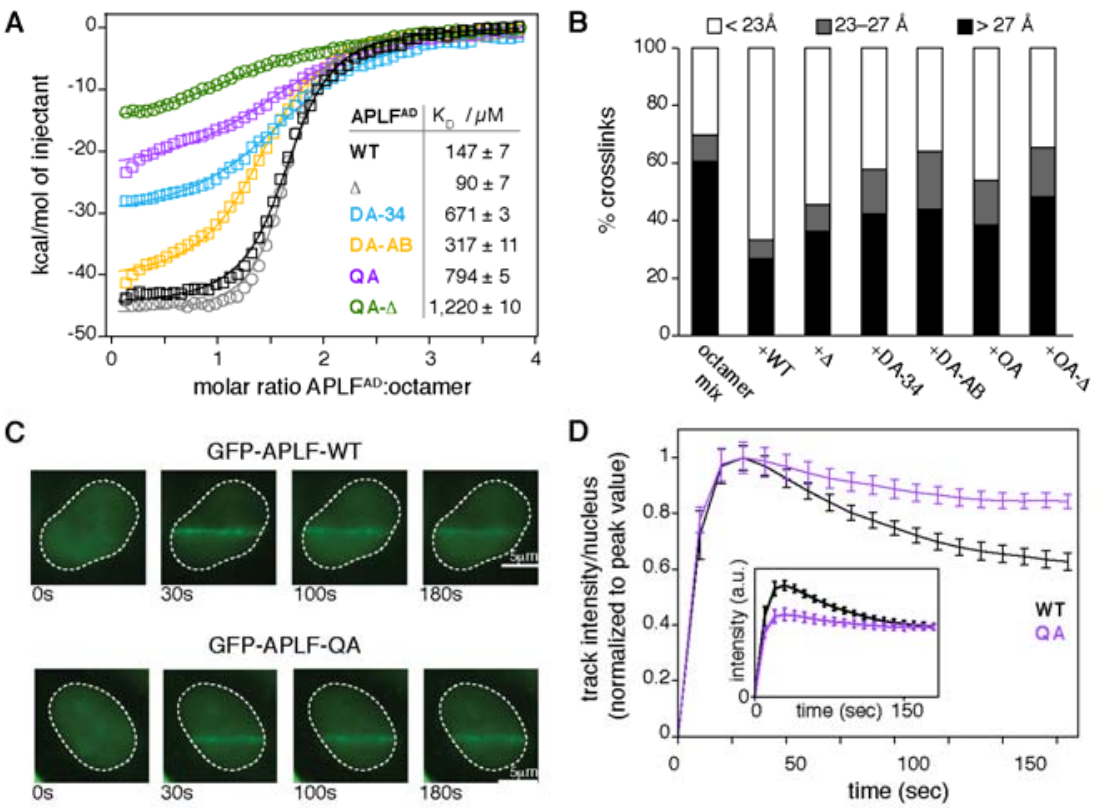

Fig. 3 | APLF $^{\mathrm{AD}}$ aromatic anchor residues are required for assembly, chaperone activity and efficient DNA repair. A, ITC binding curves and derived affinities $\left(K_{D}\right)$ of $A P L F^{A D}$ wild-type $(W T)$, the truncation mutant used for crystallization $\left(\mathrm{APLF}^{\mathrm{AD}-\Delta}, \Delta\right)$ or the double $(\mathrm{Y} 462 \mathrm{~A} / \mathrm{F} 468 \mathrm{~A}=\mathrm{DA}-34 ; \mathrm{Y} 476 \mathrm{~A} / \mathrm{W} 485 \mathrm{~A}=\mathrm{DA}-\mathrm{AB})$ and quadruple (Y462A/F468A/Y476A/W485A = QA) mutants titrated to octamer-mix. B, Percentage of octamer-compatible and -incompatible histone-histone lysine cross-links based on surface accessible $C \alpha-C \alpha$ distances in the nucleosomal structure (PDB:2PYO) identified by XL-MS. C, Live cell imaging of the recruitment of eGFP-APLF to $365 \mathrm{~nm}$ UV-A laser micro-irradiation induced DNA damage tracks in BrdU-sensitized U2OS cells transfected with the indicated plasmids. Representative images are shown. Scale bars: $5 \mu \mathrm{m}$. D, Normalized quantification of the recruitment and release of eGFP-APLF to DNA damage tracks, showing that the QA mutation causes retention of APLF at the damage site. Unnormalized data in the inset. Data represent the mean values \pm standard error of the mean from 128 (WT) or 130 (QA mutant) cells acquired in 5 independent experiments.

Having established that $\mathrm{APLF}^{\mathrm{AD}}$ can chaperone the histone octamer, we asked whether APLF ${ }^{\mathrm{AD}}$ may also be able to promote deposition of the octamer on DNA to form nucleosomes. First, we used a precipitation-rescue assay $(12,26)$ to test if $\operatorname{APLF}^{\mathrm{AD}}$ prevents non-native histone-DNA contacts, as expected for a histone chaperone(1). Indeed, APLF $^{A D}$ rescued the precipitation of histones on DNA in a way that depends on the presence of the key anchor aromatic residues (Fig. 4A). This demonstrates that $\mathrm{APLF}^{\mathrm{AD}}$ functions as a bona fide histone chaperone using the binding mode observed in the crystal structure. Next, we tested whether APLF ${ }^{A D}$ facilitates nucleosome formation. Using the nucleosome assembly and quantitation (NAQ) assay(27), we monitored nucleosome formation upon incubation of the octamer-mix with $207 \mathrm{bp}$ DNA fragments in the presence of $\mathrm{APLF}^{\mathrm{AD}}$, followed by digestion with micrococcal nuclease (MNase). Addition of APLF ${ }^{\mathrm{AD}}$ to the histones 
caused increased protection of DNA fragments of 125-160 bp in a dose-dependent manner, consistent with nucleosome formation (Fig. 4B,D and Fig. S17). Together, these data indicate that APLF $^{A D}$ prevents spurious histone-DNA interactions and allows deposition of the histone octamer on DNA to form nucleosomes.

We next sought to understand how APLF $^{\mathrm{AD}}$ may deposit octamers on DNA. As APLF ${ }^{\mathrm{AD}}$ does not cover the entire DNA binding surface of the histone octamer (Fig. 2), we hypothesized that the APLF ${ }^{A D}$ octamer complex would be able to bind a short piece of DNA, forming a ternary APLF ${ }^{A D}$-octamerDNA complex representing a reaction intermediate during octamer deposition. Using fluorescently labeled proteins and cross-linking to trap transient complexes, we confirmed that APLF ${ }^{\mathrm{AD}}$ alone does not bind DNA (Fig. 4D,E lane 2 vs. 3), while addition of a 49-bp DNA fragment to the histone octamer-mix alone resulted in precipitation (Fig. 4D,E lane 9). Upon incubation of the $\mathrm{APLF}^{\mathrm{AD}}$-histone octamer complex with the DNA fragment, we detected a ternary complex containing DNA, histone octamer, and $\mathrm{APLF}^{\mathrm{AD}}$ (Fig. 4D,E marked band in lane 4 and 6 and Fig. S18). Importantly, this ternary complex is not formed when using the $\mathrm{APLF}^{\mathrm{AD}}$ QA mutant (Fig. 4D,E lane 8), indicating that in absence of proper $\mathrm{APLF}^{\mathrm{AD}}$-histone binding the intermediate cannot be formed. Moreover, in the presence of a longer 147-bp DNA fragment the ternary complex could not be detected, but only DNA-histone complexes (Fig. S19), in line with the octamer being deposited on this longer DNA (as in Fig. $4 \mathrm{~B}$ ) and $A P L F^{A D}$ leaving the nucleosome product. Therefore, the $\mathrm{APLF}^{\mathrm{AD}}$-octamer-DNA complex isolated with a short DNA fragment may represent an intermediate in the octamer deposition process by APLF. 
A

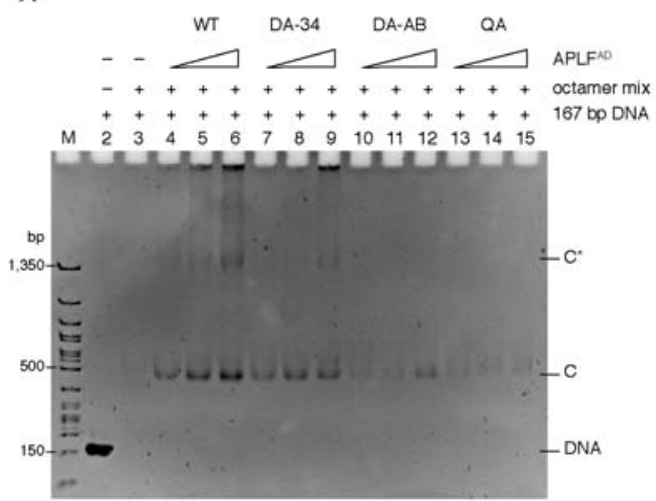

C

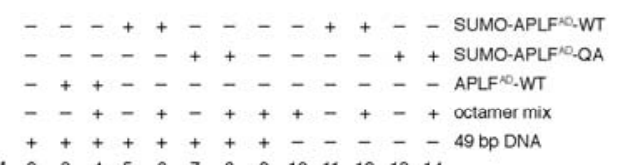

$\begin{array}{llllllllllllll}M & 2 & 3 & 4 & 5 & 6 & 7 & 8 & 9 & 10 & 11 & 12 & 13 & 14\end{array}$

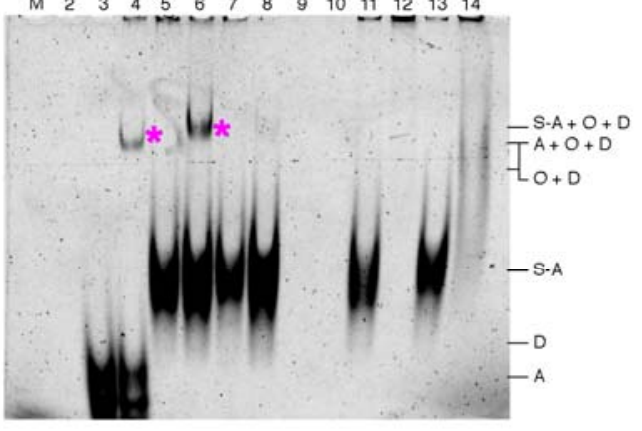

E

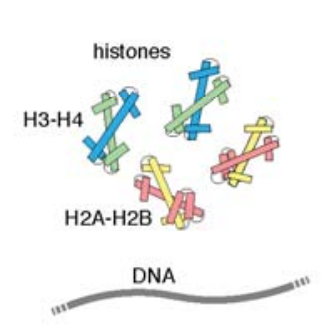

B
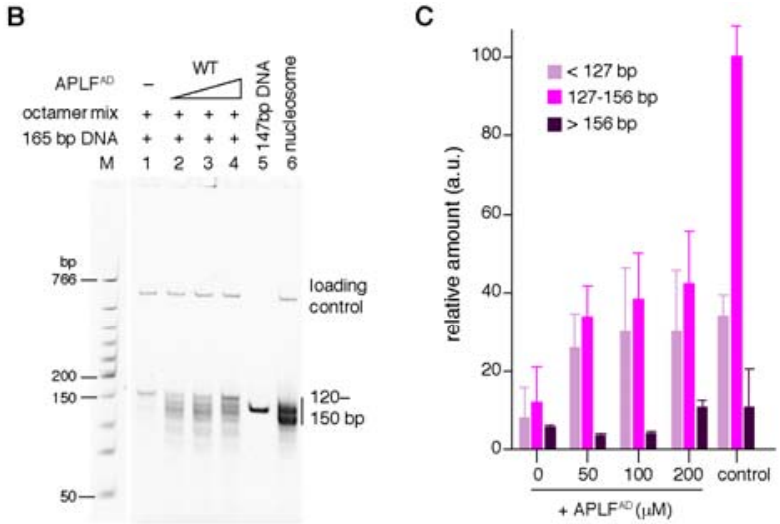

D

D $\quad-\quad-\ldots+\ldots+\ldots++-$ SUMO-APLFOWW

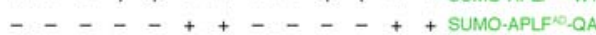

$-+\ldots+\ldots+\ldots$ - $+\ldots$ - APLFOPT

-++-+-+++-+-+ octamer mix

$++++++++---39 \mathrm{bp}$ DNA
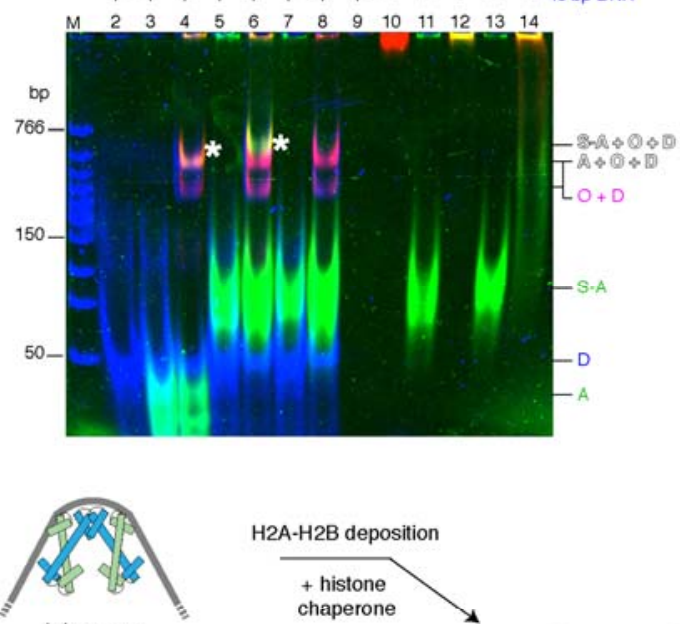

H2A-H2B deposition

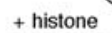

tetrasome chaperone

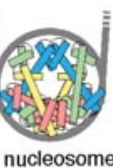

Fig. 4 | APLF ${ }^{A D}$ chaperones the histone octamer to promote nucleosome assembly. A, Native PAGE analysis of precipitation-rescue assay showing formation of soluble protein-DNA complexes (bands ' $C^{\prime}$ and ' $C^{* \prime}$ ) upon addition of increasing amounts of WT APLF ${ }^{\mathrm{AD}}$ to octamer-mix with DNA, which is strongly reduced for mutant $\mathrm{APLF}^{\mathrm{AD}}$. Band ' $\mathrm{C}$ ' corresponds to the electrophoretic mobility of nucleosomes (Fig. S14). B,C NAQ results showing MNase digestion products obtained for octamer-mix with DNA and increasing amounts of APLF ${ }^{\mathrm{AD}}$. Quantification of DNA digestion products (average and standard error of the mean $(n=3)$ ) in panel C. APLF ${ }^{\mathrm{AD}}$ increases the protected nucleosomal bands (127-156bp) ( $p=0.018,0.018,0.016$ for 50, 100, $200 \mu \mathrm{M})$ according to a one-tailed Students' $t$-test. Salt-assembled nucleosomes are used as control. D,E Native PAGE analysis of indicated mixtures of APLF $^{A D}$, octamer-mix and DNA, crosslinked with DSS. Panel D shows the Cy3scan with $\mathrm{APLF}^{\mathrm{AD}}$ signal before DNA staining, panel $\mathbf{E}$ shows a merged image of the APLF ${ }^{\mathrm{AD}}$ (green), histone 
(red) and DNA (blue) scans (see Fig. S18 for individual scans). APLF ${ }^{A D}$ forms a ternary complex with histones and DNA (asterisk in lane 3 and 6). SUMO-APLF ${ }^{A D}$ was used as control to shift the ternary band above the

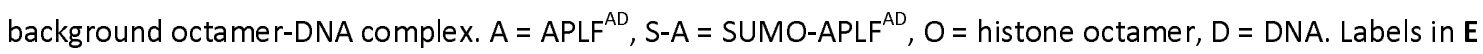
are color-coded according to the fluorescent dye. F, Model of the proposed histone chaperone mechan ism by APLF. APLF ${ }^{\mathrm{AD}}$ assembles $\mathrm{H} 2 \mathrm{~A}-\mathrm{H} 2 \mathrm{~B}$ and $\mathrm{H} 3-\mathrm{H} 4$ simultaneously in an octameric configuration, and deposits them on DNA using a transient ternary intermediate. This contrasts with the stepwise nucleosome assembly where $(\mathrm{H} 3-\mathrm{H} 4)_{2}$ deposition precedes $\mathrm{H} 2 \mathrm{~A}-\mathrm{H} 2 \mathrm{~B}$.

Together, these data lead to a compelling model for nucleosome formation by APLF ${ }^{A D}$ that contrasts sharply with the stepwise nucleosome assembly pathway used by the other ATP-independent histone chaperones characterized so far(28) (Fig. 4F). Our data showed that APLF ${ }^{A D}$, as a flexible and disordered protein, can bind both $\mathrm{H} 2 \mathrm{~A}-\mathrm{H} 2 \mathrm{~B}$ and $\mathrm{H} 3-\mathrm{H} 4$ simultaneously, tethering them into a histone octamer in its nucleosomal configuration. In the complex, APLF ${ }^{A D}$ stabilizes the histone octamer and prevents non-native histone-DNA interactions. We propose that the exposed histone dyad region, where the octamer has highest affinity for DNA(29) and where DNA binding stabilizes the H3-H3' interface of the $(\mathrm{H} 3-\mathrm{H} 4)_{2}$ tetramer, allows the interaction with DNA to initiate octamer deposition. The DNA may then displace $\mathrm{APLF}^{\mathrm{AD}}$ as it wraps around the histone octamer to form the nucleosome (Fig. 4F). Notably, two copies of APLF are present at DNA break sites(30), suggesting that the acidic domain of APLF could provide the NHEJ machinery with the capacity to assemble or capture histone octamers, store them during the DNA repair process, and to promote nucleosome assembly to restore chromatin after repair.

By tethering $\mathrm{H} 2 \mathrm{~A}-\mathrm{H} 2 \mathrm{~B}$ and $\mathrm{H} 3-\mathrm{H} 4$ in their native nucleosomal configuration, APLF adds a remarkable new mode of action in the repertoire of histone chaperones. Our data demonstrates that histone octamer assembly can be uncoupled from nucleosome assembly and can be controlled by a single histone chaperone. Interestingly, while many histone chaperones contain acidic stretches(31), sequence analysis based on the presence of the key aromatic anchor residues revealed no obvious candidates for a chaperone with similar histone octamer chaperone activity as APLF ${ }^{A D}$ (Fig. S20). It will be interesting to see if other histone chaperones may retain this function and how this is regulated. We speculate that chaperoning of octamers may be a more widespread mechanism to maintain $\mathrm{H} 3-\mathrm{H} 4$ as well as $\mathrm{H} 2 \mathrm{~A}-\mathrm{H} 2 \mathrm{~B}$ with their modifications and variants within the same nucleosome, during temporary chromatin disassembly throughout the genome. 


\section{References}

1. R. A. Laskey, B. M. Honda, A. D. Mills, J. T. Finch, Nucleosomes are assembled by an acidic protein which binds histones and transfers them to DNA. Nature. 275, 416-420 (1978).

2. C. M. Hammond, C. B. Strømme, H. Huang, D. J. Patel, A. Groth, Histone chaperone networks shaping chromatin function. Nat. Rev. Mol. Cell Biol. 18, 141-158 (2017).

3. Z. A. Gurard-Levin, J.-P. Quivy, G. Almouzni, Histone chaperones: Assisting histone traffic and nucleosome dynamics. Annu. Rev. Biochem. 83, 487-517 (2014).

4. J. A. Kleinschmidt, A. Seiter, H. Zentgraf, Nucleosome assembly in vitro: Separate histone transfer and synergistic interaction of native histone complexes purified from nuclei of Xenopus laevis oocytes. EMBO J. 9, 1309-1318 (1990).

5. S. J. Elsässer, S. D'Arcy, Towards a mechanism for histone chaperones. Biochim. Biophys. Acta - Gene Regul. Mech. 1819, 211-221 (2012).

6. N. Iles, S. Rulten, S. F. El-Khamisy, K. W. Caldecott, APLF (C2orf13) is a novel human protein involved in the cellular response to chromosomal DNA strand breaks. Mol. Cell. Biol. 27, 3793-3803 (2007).

7. C. J. Macrae, R. D. McCulloch, J. Ylanko, D. Durocher, C. A. Koch, APLF (C2orf13) facilitates nonhomologous end-joining and undergoes ATM-dependent hyperphosphorylation following ionizing radiation. DNA Repair (Amst). 7, 292-302 (2008).

8. G. J. Grundy, S. L. Rulten, Z. Zeng, R. Arribas-Bosacoma, N. Iles, K. Manley, A. Oliver, K. W. Caldecott, APLF promotes the assembly and activity of non-homologous end joining protein complexes. EMBO J. 32, 112-125 (2013).

9. L. Woodbine, A. R. Gennery, P. A. Jeggo, The clinical impact of deficiency in DNA nonhomologous end-joining. DNA Repair (Amst). 16, 84-96 (2014).

10. B. J. Sishc, A. J. Davis, The role of the core non-homologous end joining factors in carcinogenesis and cancer. Cancers (Basel). 9 (2017).

11. P. V. Mehrotra, D. Ahel, D. P. Ryan, R. Weston, N. Wiechens, R. Kraehenbuehl, T. OwenHughes, I. Ahel, DNA repair factor APLF is a histone chaperone. Mol. Cell. 41, 46-55 (2011).

12. I. Corbeski, K. Dolinar, H. Wienk, R. Boelens, H. Van Ingen, DNA repair factor APLF acts as a $\mathrm{H} 2 \mathrm{~A}-\mathrm{H} 2 \mathrm{~B}$ histone chaperone through binding its DNA interaction surface. Nucleic Acids Res. 46, 7138-7152 (2018). 
13. S. Dutta, I. V. Akey, C. Dingwall, K. L. Hartman, T. Laue, R. T. Nolte, J. F. Head, C. W. Akey, The crystal structure of nucleoplasmin-core: Implications for histone binding and nucleosome assembly. Mol. Cell. 8, 841-853 (2001).

14. K. F. Tóth, J. Mazurkiewicz, K. Rippe, Association states of nucleosome assembly protein 1 and its complexes with histones. J. Biol. Chem. 280, 15690-15699 (2005).

15. S. Muto, M. Senda, Y. Akai, L. Sato, T. Suzuki, R. Nagai, T. Senda, M. Horikoshi, Relationship between the structure of SET/TAF-I $\beta /$ INHAT and its histone chaperone activity. Proc. Natl. Acad. Sci. U. S. A. 104, 4285-4290 (2007).

16. Y. Tsunaka, Y. Fujiwara, T. Oyama, S. Hirose, K. Morikawa, Integrated molecular mechanism directing nucleosome reorganization by human FACT. Genes Dev. 30, 673-686 (2016).

17. M. Zhang, H. Liu, Y. Gao, Z. Zhu, Z. Chen, P. Zheng, L. Xue, J. Li, M. Teng, L. Niu, Structural insights into the association of Hif1 with histones $\mathrm{H} 2 \mathrm{~A}-\mathrm{H} 2 \mathrm{~B}$ dimer and $\mathrm{H} 3-\mathrm{H} 4$ tetramer. Structure. 24, 1810-1820 (2016).

18. Y. Lorch, M. Zhang, R. D. Kornberg, Histone octamer transfer by a chromatin-remodeling complex. 96, 389-392 (1999).

19. C. E. Rowe, G. J. Narlikar, The ATP-dependent remodeler RSC transfers histone dimers and octamers through the rapid formation of an unstable encounter intermediate. Biochemistry. 49, 9882-9890 (2010).

20. J. Markert, K. Zhou, K. Luger, Smarcad1 is an ATP-dependent histone octamer exchange factor with de novo nucleosome assembly activity. Sci. Adv. 7, 1-12 (2021).

21. K. Luger, A. W. Mäder, R. K. Richmond, D. F. Sargent, T. J. Richmond, Crystal structure of the nucleosome core particle at 2.8 Å resolution. Nature. 389, 251-260 (1997).

22. C. A. Davey, D. F. Sargent, K. Luger, A. W. Maeder, T. J. Richmond, Solvent mediated interactions in the structure of the nucleosome core particle at $1.9 \AA$ resolution. J. Mol. Biol. 319, 1097-1113 (2002).

23. D. J. Kemble, L. L. McCullough, F. G. Whitby, T. Formosa, C. P. Hill, FACT disrupts nucleosome structure by binding H2A-H2B with conserved peptide motifs. Mol. Cell. 60, 294-306 (2015).

24. Y. Wang, S. Liu, L. Sun, N. Xu, S. Shan, F. Wu, X. Liang, Y. Huang, E. Luk, C. Wu, Z. Zhou, Structural insights into histone chaperone Chz1-mediated H2A.Z recognition and histone replacement. PLOS Biol. 17, 1-20 (2019). 
25. M. D. Ricketts, J. Han, M. R. Szurgot, R. Marmorstein, Molecular basis for chromatin assembly and modification by multiprotein complexes. Protein Sci. 28, 329-343 (2019).

26. M. Hondele, T. Stuwe, M. Hassler, F. Halbach, A. Bowman, E. T. Zhang, B. Nijmeijer, C. Kotthoff, V. Rybin, S. Amlacher, E. Hurt, A. G. Ladurner, Structural basis of histone H2A-H2B recognition by the essential chaperone FACT. Nature. 499, 111-114 (2013).

27. F. Mattiroli, Y. Gu, K. Luger, Measuring nucleosome assembly activity in vitro with the nucleosome assembly and quantification (NAQ) assay. Bio-Protocol. 8, 1-11 (2018).

28. S. Smith, B. Stillman, Stepwise assembly of chromatin during DNA replication in vitro. EMBO J. 10, $971-980$ (1991).

29. M. A. Hall, A. Shundrovsky, L. Bai, R. M. Fulbright, J. T. Lis, M. D. Wang, High-resolution dynamic mapping of histone-DNA interactions in a nucleosome. Nat. Struct. Mol. Biol. 16, 124-129 (2009).

30. M. Hammel, Y. Yu, S. K. Radhakrishnan, C. Chokshi, M. S. Tsai, Y. Matsumoto, M. Kuzdovich, S. G. Remesh, S. Fang, A. E. Tomkinson, S. P. Lees-Miller, J. A. Tainer, An intrinsically disordered APLF links Ku, DNA-PKCs, and XRCC4-DNA ligase IV in an extended flexible non-homologous end joining complex. J. Biol. Chem. 291, 26987-27006 (2016).

31. C. Warren, D. Shechter, Fly fishing for histones: Catch and release by histone chaperone intrinsically disordered regions and acidic stretches. J. Mol. Biol. 429, 2401-2426 (2017). 


\section{Acknowledgements}

We thank Raymond Schellevis for assistance in protein purification, Gert Folkers for lab support, and Johan van der Zwan and Andrei Gurinov for support and maintenance of the NMR infrastructure (all from the Utrecht NMR Group at Utrecht University). We thank Patrick Celie and Alexander Fish for help in setting up crystallization screens and Tatjana Heidebrecht for fishing crystals (all from Netherlands Cancer Institute). We thank the Swiss Light Source staff for X-ray diffraction synchrotron access and support. We thank the beamline P12 staff of EMBL Hamburg and the PETRA III storage ring staff of DESY (Hamburg, Germany) for SAXS synchrotron access. We thank Anneloes Blok and Marcellus Ubbink from Macromolecular Biochemistry, Leiden Institute of Chemistry, Leiden University for providing access to the VP-ITC MicroCalorimeter. We thank Pamela Dyer and Karolin Luger from the Department of Biochemistry, University of Colorado at Boulder for providing plasmids of tailless histones $\mathrm{H} 3$ and $\mathrm{H} 4$.

\section{Funding}

This work was supported by the Marie Curie Initial Training Network Innovative Doctoral Programme ManiFold (grant 317371; EC-FP7/2007-201); the Netherlands Organisation for Scientific Research (grants 723.013.010 to $\mathrm{Hvl}, 175.107 .301 .10$ and 700.53.103 to RB and National Roadmap grant 184.032.207 to uNMR-NL, the National Roadmap Large-Scale NMR Facility of the Netherlands); an ERC starting grant to FM (grant 851564); Instruct-ULTRA (grant 731005), an EU H2020 project to further develop the services of Instruct-ERIC; and the EU Horizon 2020 program INFRAIA project Epic-XS (project 823839). Financial support for SAXS measurements at beamline P12 operated by EMBL Hamburg at the PETRA III storage ring (DESY, Hamburg, Germany) was provided under the framework of the Horizon 2020 Programme of the European Union through iNEXT (grant 653706) and iNEXT-Discovery (grant 871037).

\section{Author contributions}

I.C. performed experiments and contributed to all aspects of the study; in particular, protein production and purification including cloning, mutagenesis, binding studies by SEC, ITC and NMR, chaperone activity assay, crystallization, data analysis, and interpretation. X.G. performed crystallization, collected diffraction data, and solved the crystal structure. B.V.E and F.M. performed the nucleosome assembly and ternary intermediate assay and data analysis. D.F. performed native and cross-linking mass spectrometry and data analysis. M.A.G performed SAXS experiments and 
data analysis. W.W. and K.V. constructed transfection vectors and performed cellular DNA damage assays. H.W. provided expertise in NMR experiments. H.v.A. provided expertise in cellular DNA damage repair experiments and data interpretation. D.S. provided expertise in SAXS experiments and data interpretation. A.J.H. provided expertise in mass spectrometry experiments and data interpretation. R.B. provided NMR expertise and supervision. T.K.S. provided crystallography expertise and data interpretation. F.M. provided expertise in nucleosome assembly experiments and data interpretation. H.v.I. and R.B. secured funding. H.v.l. performed NMR experiments, data analysis and provided supervision. I.C., F.M and H.v.I. wrote the manuscript and prepared the figures with input from all co-authors. All authors reviewed the manuscript and contributed to the discussion of the results.

\section{Competing interests}

None of the authors declare a competing interest.

\section{Data availability}

The coordinates of the $\mathrm{APLF}^{\mathrm{AD}}$-histone octamer complex have been deposited in the Protein Data Bank under accession number 6YN1. The experimental SAXS data and models are deposited in SASBDB with the accession codes: SASDJJ5. The Native MS and cross-linking mass spectrometry data are available via Figshare: https://figshare.com/s/43e763c4f078ce450cf8.

\section{Supplementary Materials}

Materials and Methods

Figs. S1 to S20

Tables S1 to S3 